\title{
Dietary patterns including fruits, vegetables, whole grains, low fat dairy, and lean meats lowered mortality risk in women
}

Kant AK, Schatzkin A, Graubard BI, et al. A prospective study of diet quality and mortality in women. JAMA 2000 Apr 26;283:2109-15.

QUESTION: Is overall diet quality associated with mortality in women?

Design

Cohort study of women in the Breast Cancer Detection and Demonstration Project (BCDDP) with a median follow up of 5.6 years.

Setting

27 cities in the US.

Participants

42254 women (mean age 61 y, $87 \%$ white).

\section{Assessment of risk factors}

Women completed a 62 item food frequency questionnaire by post, which collected information on the frequency and portion sizes of foods consumed over the previous year. Overall diet quality was assessed by the Recommended Foods Score (RFS), which was the sum of 23 foods (consumed at least once a week; maximum score 23) that were recommended in current dietary guidelines (fruits, vegetables, whole grains, low fat dairy, lean meats, and poultry). For analysis, women were divided into quartiles based on their RFS; the lowest quartile was given a relative risk (RR) of 1.0. Data were adjusted for age; race; education level; body mass index; smoking status; alcohol use; energy intake; history of cancer, heart disease, or diabetes; menopausal hormone use; and physical activity.

\section{Main outcome measures}

All cause and cause specific mortality. When follow up data were not available, the National Death Index was searched.

\section{Main results}

2065 deaths from all causes occurred. The RFS (mean 11.4) was positively associated with intake of energy and protein, percentage of energy from carbohydrates, and micronutrient intake $(p<0.05)$ and inversely associated with percentage of energy from fat $(\mathrm{p}<0.05)$. Multivariate analyses showed an association between RFS and risk of death from all causes ( $p$ for trend $<0.001$ ), all sites cancer ( $\mathrm{p}$ for trend $<0.001$ ), coronary artery disease ( $\mathrm{p}$ for trend 0.03 ), and stroke ( $\mathrm{p}$ for trend 0.02 ) (table).

\section{Conclusion}

Consumption of foods that are recommended in current dietary guidelines, including fruits, vegetables, whole grains, low fat dairy, and lean meats and poultry was associated with a decreased risk of all cause and cause specific mortality in women.
Multivariate relative risks (RRs) of mortality for the highest quartile of Recommended Foods Score (RFS)*

\begin{tabular}{ll}
$\begin{array}{l}\text { Outcomes at a median of } 5.6 \\
\text { years }\end{array}$ & RR $(95 \% \mathrm{Cl})$ \\
\hline Death from all causes & $0.69(0.61$ to 0.78$)$ \\
\hline Death from all sites cancer & $0.60(0.49$ to 0.74$)$ \\
\hline Death from coronary artery disease & $0.67(0.47$ to 0.95$)$ \\
\hline Death from stroke & $0.58(0.35$ to 0.96$)$ \\
\hline $\begin{array}{l}\text { *The reference group was the lowest quartile of RFS. Adjusted for age; race; } \\
\text { education level; body mass index; smoking status; alcohol use; energy intake; } \\
\text { history of cancer, heart disease, or diabetes; menopausal hormone use; and } \\
\text { physical activity. }\end{array}$
\end{tabular}

\section{COMMENTARY}

This well designed, multisite, prospective cohort study by Kant $e t$ al provides an important contribution to the diet and health promotion literature. A large population of older women were included, and the relation between diet quality (measured by a food frequency questionnaire) and mortality risk was examined. This same approach has been used successfully in a clinical study of diet quality and blood pressure. $^{1}$

This study cannot prove or disprove the importance of diet quality because it is not a randomised controlled trial; however, it does provide additional evidence of the importance of following the recommended American guidelines for intake of fruits, vegetables, whole grains, lean meat, and low fat dairy products. These relations were statistically significant for risk of both all cause and disease specific mortality.

For data analysis, the women were divided into 4 quartiles based on their RFS. In general, comparisons among the 4 groups showed consistent increases in energy intake, dietary fibre, vitamins, and micronutrients and a reduction in percentage of energy from fat. This is particularly interesting for clinicians because the results showed a non-linear relation with the greatest decline in the risk of mortality occurring between the 2 groups with the lowest RFS. This suggests that patients making relatively small dietary changes may also reduce their mortality risk or it suggests that diet quality serves as a clinical proxy for other factors associated with healthy lifestyle, such as smoking (eg, people who eat better also avoid smoking). These diet mortality relations are complicated, but this study provides further evidence for counselling patients about the relation between a good diet and health.

George F Shuster, RN, DNS Associate Professor of Nursing University of New Mexico Albuquerque, New Mexico, USA

1 Appel LJ, Moore TJ, Obarzanek E, et al. A clinical trial of the effects of dietary patterns on blood pressure. DASH Collaborative Research Group. NEngl J Med 1997;336:111724. 